\title{
Laparoscopic Colectomy
}

National Cancer Institute

\section{Source}

National Cancer Institute. Laparoscopic Colectomy. NCI Thesaurus. Code C15762.

Surgical removal of part or all of the colon, using a laparoscope. 